\title{
Nebulized Magnesium Sulfate for Treatment of Persistent Pulmonary Hypertension of Newborn: A Pilot Randomized Controlled Trial
}

\author{
Elsayed Abdelkreem ${ }^{1}$ (D) Shaimaa M. Mahmoud ${ }^{1} \cdot$ Moustafa O. Aboelez $^{2} \cdot$ Mohamed Abd El Aal $^{1}$
}

Received: 19 August 2020 / Accepted: 23 December 2020 / Published online: 8 January 2021

(C) Dr. K C Chaudhuri Foundation 2021

\begin{abstract}
Objectives To investigate the effectiveness of nebulized magnesium sulfate in treating persistent pulmonary hypertension of newborn (PPHN).

Methods Twenty-eight mechanically ventilated term neonates with severe PPHN were randomized into two groups: NebMag group $(n=14)$, who receiving nebulized isotonic magnesium $(1024 \mathrm{mg} / \mathrm{h})$, and IVMag group $(\mathrm{n}=14)$, who received intravenous magnesium ( $200 \mathrm{mg} / \mathrm{kg}$ over $30 \mathrm{~min}$, followed by $50 \mathrm{mg} / \mathrm{kg} / \mathrm{h}$ ). The study time frame was $24 \mathrm{~h}$. Outcome measures were the changes in oxygenation index (OI), mean arterial blood pressure (MABP), vasoactive inotropic score (VIS), and serum magnesium level.

Results Baseline demographic, ventilatory, and hemodynamic characteristics were comparable between the two groups. At the end of the study, the OI decreased by $44.3 \%$ in the NebMag group compared with $35.3 \%$ in the IVMag group (mean difference $-3.14 ; 95 \%$ CI $-5.08,-1.19 ; p 0.003$ ). The NebMag group had a higher MABP (mean difference $2.29 \mathrm{mmHg} ; 95 \%$ CI 1.80, 2.77; $p 0.000$ ) and lower VIS (mean difference $-14.64 ; 95 \% \mathrm{CI}-16.52,-12.77 ; p 0.000$ ) at the 24-h study time point. The increase in serum magnesium level, measured at 12-h study time point, was lower in the NebMag group (mean difference $-2.26 \mathrm{mmol} / \mathrm{L}$; 95\% CI $-2.58,-1.96 ; p 0.000)$.

Conclusion Nebulized magnesium sulfate may be an effective therapeutic modality for neonates with severe PPHN on mechanical ventilation, but this should be confirmed by larger studies.

Retrospectively registered at www.clinicaltrials.gov (identifier: NCT04328636).
\end{abstract}

Keywords Neonate $\cdot$ Hypoxia $\cdot$ Persistent fetal circulation $\cdot$ PPHN $\cdot$ Magnesium $\cdot$ Nebulizer

\section{Introduction}

Persistent pulmonary hypertension of the newborn (PPHN) is a potentially life-threatening disease characterized by a failure of the normal postnatal circulatory transition $[1,2]$. PPHN has an incidence of $0.5-7$ per 1000 live births, accounts for 2\%$10 \%$ of admissions at neonatal intensive care unit (NICU), and

Elsayed Abdelkreem

d.elsayedmohammed@med.sohag.edu.eg

1 Department of Pediatrics, Faculty of Medicine, Sohag University, Sohag 82524, Egypt

2 Department of Pharmaceutical Medicinal Chemistry, Faculty of Pharmacy, Sohag University, Sohag, Egypt imposes significant mortality (4\%-33\%) and neurodevelopment impairment $(25 \%)[3,4]$.

Management of PPHN generally includes optimizing oxygenation, ventilation, and hemodynamics as well as pulmonary vasodilators [5, 6]. Advances in medical treatment, particularly inhaled nitric oxide (iNO), high-frequency ventilation (HFV), and extracorporeal membrane oxygenation (ECMO), have significantly improved the survival of neonates with PPHN. However, these expensive and technically demanding therapeutic options are not commonly available in resource-limited settings [7, 8]. In addition, a third of infants with PPHN show an inadequate response to iNO [4]. Therefore, studies have investigated other therapeutic options, such as phosphodiesterase inhibitors, prostanoids, endothelin antagonists, and magnesium sulfate $[5,9,10]$.

Being cheap and readily available, intravenous magnesium sulfate has been used in treating PPHN in limited-resource 
settings [7]. However clinical evidence supporting its use is currently not available [11]. Moreover, magnesium sulfate is not a selective vasodilator, and its systemic administration commonly causes systemic hypotension as well as cardiac and neurological adverse effects $[11,12]$. The authors hypothesized that delivering magnesium sulfate by nebulization may enhance effectiveness and minimizes systemic adverse effects. To the best of authors' knowledge, there have been no reports on the use of nebulized magnesium sulfate in PPHN.

The present study aimed to investigate the effectiveness of nebulized magnesium sulfate in PPHN. This is a concept-ofproof, pilot, clinical trial to evaluate the feasibility of conducting a larger multicenter study.

\section{Material and Methods}

This was a double-blinded, randomized, parallel two-arm, clinical trial, which included two groups of neonates with PPHN: the NebMag group, who received nebulized magnesium sulfate and intravenous placebo, and the IVMag group, who received intravenous magnesium sulfate and nebulized placebo. The authors conducted this pilot study between November 1, 2017 and July 1, 2020 at the neonatal intensive care unit (NICU) of Sohag University Hospital. This is a tertiary care center, which has 32 incubators and serves a population of six million with an average annual admission of 600 newborns; however, iNO, HFV, and ECMO are currently not available, and surfactant use is mostly limited to premature babies with respiratory distress syndrome.

Eligibility criteria were neonates born at $\geq 37 \mathrm{wk}$ gestation with a birth weight of 2.5-4 kg and postnatal age of 6-72 h. Neonates had to be connected to mechanical ventilation with an oxygenation index $(\mathrm{OI})>30$ on two occasions at least 15 min apart. The authors included only newborns with documented PPHN as confirmed by echocardiography using Vivid T2 Pro (GE Medical systems [China] Co. Ltd., Jiangsu, China) and $8 \mathrm{MHz}$ transducer. The echocardiogram had to show a predominant right-to-left or bidirectional shunt (through ductus arteriosus and/or foramen ovale) and/or tricuspid regurgitant jet with a pressure gradient $\geq 2 / 3$ of the systemic systolic blood pressure $[4,13]$.

Exclusion criteria were failure to obtain informed consent; infants of mothers who received magnesium sulfate within $48 \mathrm{~h}$ before labor; congenital heart diseases other than patent ductus arteriosus (PDA) and foramen ovale; major congenital anomalies, including congenital diaphragmatic hernia and lung hypoplasia; prior need for cardiopulmonary resuscitation; mean arterial blood pressure (MABP) $<35 \mathrm{mmHg}$ despite therapy with volume infusions and vasoactive inotropes; impaired kidney function; and prior administration of pulmonary vasodilators.
The authors prepared a magnesium sulfate $10 \%$ solution for intravenous administration by diluting an intravenous form of magnesium sulfate heptahydrate 50\% (Memphis Co., Cairo, Egypt) with glucose 5\% (Allmed Middle East Co., Giza, Egypt). Pharmacy designed $60 \mathrm{~mL}$ aliquots of magnesium sulfate $10 \%$ and similar aliquots of glucose $5 \%$ as a placebo (to be discarded $24 \mathrm{~h}$ after preparation). Study participants received either active drug or placebo through an intravenous line in a loading dose of $2 \mathrm{~mL} / \mathrm{kg}$ over $30 \mathrm{~min}$ (equivalent to magnesium sulfate $200 \mathrm{mg} / \mathrm{kg}$ in case of the active drug), followed by a continuous infusion at a rate of $0.5 \mathrm{~mL} /$ $\mathrm{kg} / \mathrm{h}$ (equivalent to magnesium sulfate $50 \mathrm{mg} / \mathrm{kg} / \mathrm{h}$ in case of the active drug) for the $24 \mathrm{~h}$ study duration.

For nebulization, the authors prepared an isotonic solution of magnesium sulfate $(64 \mathrm{mg} / \mathrm{mL}, 265 \mathrm{mmol} / \mathrm{L}, 306 \mathrm{mOsm} / \mathrm{L})$ by diluting an intravenous form of magnesium sulfate heptahydrate $10 \%$ with distilled sterile water (Allmed Middle East Co.). Pharmacy designed 4-ml aliquots of isotonic magnesium sulfate $(256 \mathrm{mg}$ ) and similar aliquots of isotonic saline (Allmed Middle East Co.) as a placebo (to be discarded $24 \mathrm{~h}$ after preparation). During the 24-h study duration, a 4-mL aliquot of active drug or placebo was loaded every $15 \mathrm{~min}$ to the jet nebulizer of Babylog 8000 plus ventilator (Dräger Medical Inc., Lübeck, Germany). The authors placed the nebulizer in the inspiratory limb, $20 \mathrm{~cm}$ away from the $\mathrm{Y}$ piece, of a neonatal heated-wired silicone rubber breathing circuit (Dräger Medical Inc.). The nebulizer was operated by pressurized medical air from the ventilator $(2 \mathrm{~L} / \mathrm{min})$ with a tested nebulization efficiency of approximately $4 \mathrm{~mL} / 15 \mathrm{~min}$. The healthcare providers visually inspected the reservoir of nebulizer before each loading for any remaining volume. The respiratory humidifier (Fisher \& Paykel Healthcare Ltd., Auckland, New Zealand) was set at $37{ }^{\circ} \mathrm{C}$.

Enrolled newborns were randomized into two equal-sized groups using computer-generated numbers (randomization. com), which were sealed in sequentially numbered opaque envelopes. When the parents of an eligible newborn consent to enter the trial, the physicians opened an envelope and started the allocated treatment. Each neonate was assigned a unique identification number. Pharmacy filled the active and placebo preparations for nebulized and intravenous administration in similar containers with sealed codes for identification. Participants' families, treating clinicians, nurses, echocardiographers, data collectors, and statisticians were unaware of the randomization method, group assignment, and drug/placebo therapy.

All study newborns were connected to Babylog 8000 plus ventilator using pressure control-synchronized intermitted mandatory ventilation with volume guarantee as the standard mode of ventilation. The treating physicians adjusted the ventilator flow to compensate for additional airflow during nebulization. The management aimed to achieve adequate oxygenation and ventilation targeting a preductal peripheral capillary 
oxygen saturation $\left(\mathrm{SpO}_{2}\right)$ of $92 \%-97 \%$, preductal partial pressure of oxygen in arterial blood $\left(\mathrm{PaO}_{2}\right)$ of $50-80 \mathrm{mmHg}$, postductal $\mathrm{SpO}_{2}$ of $>70 \%$, $\mathrm{pH}$ of 7.3-7.45, and partial pressure of carbon dioxide in arterial blood $\left(\mathrm{PaCO}_{2}\right)$ of $45-$ $55 \mathrm{mmHg}$. None of the studied newborns received surfactant therapy. Newborns received vasoactive inotropes (dopamine 10-20 $\mu \mathrm{g} / \mathrm{kg} / \mathrm{min}$, dobutamine 10-20 $\mu \mathrm{g} / \mathrm{kg} / \mathrm{min}$, epinephrine $0.05-1 \mu \mathrm{g} / \mathrm{kg} / \mathrm{min}$ ) and volume expanders $(10-15 \mathrm{~mL} / \mathrm{kg}$ normal saline in case of intravascular volume depletion) to keep systemic blood pressure at the higher normal range [7]. Other general management included optimizing temperature, sedation, electrolytes, glucose, and hemoglobin as well as treatment of the underlying condition, if possible. Neonates underwent gradual weaning from mechanical ventilation according to a standard protocol as soon as they achieve stable values of the above-mentioned oxygenation, ventilation, and hemodynamic targets; however, none was extubated during the 24-h study time frame.

Treating physicians followed the patient's hemodynamics by Vista 120 monitor (Dräger Medical Inc.,), including $\mathrm{SpO}_{2}$ in the right arm (preductal) and right leg (postductal) and MABP. The fraction of inspired oxygen $\left(\mathrm{FiO}_{2}\right)$, mean airway pressure (MAP), and other ventilatory parameters were recorded from Babylog 8000 plus ventilator. Postductal arterial blood samples were drawn before (baseline) and 2, 6, 12, and $24 \mathrm{~h}$ following study drug administration for gas analysis by ABL800 FLEX blood gas analyzer (Radiometer Medical ApS, Bronshoj, Denmark). The authors measured serum magnesium level before and $12 \mathrm{~h}$ following study drug administration.

The primary outcome in the present study was the change in oxygenation index (OI) from baseline to 2,6 , 12 , and $24 \mathrm{~h}$ following study drug administration [1]. The authors preferred using OI, which considers both oxygenation status and ventilatory support, over merely reporting individual values of $\mathrm{SpO}_{2}, \mathrm{PaO}_{2}, \mathrm{FiO}_{2}$, and ventilatory parameters. The secondary outcomes were the changes in (a) MABP and vasoactive inotropic score (VIS) from baseline to $2,6,12$, and $24 \mathrm{~h}$ following study drug administration [14]; and (b) serum magnesium level from baseline to $12 \mathrm{~h}$ after study drug administration.

Data were analyzed using IBM SPSS $®$ software for Windows version 20 (IBM Corp., Armonk, NY, USA). The authors compared variables between the two study groups using Student's t test (continuous variables) and Fisher exact test (qualitative variables). Repeated measures ANOVA was used for comparing serial measures of OI, MAPB, and VIS within and between groups. A $p$ value (two-tailed) $<0.05$ was considered statistically significant.

This study was approved by the Research Ethics Committee of Faculty of Medicine, Sohag University, and was carried out following the principles contained within the 1964 Declaration of Helsinki and as revised in 2013. Informed consent was obtained from parents or authorized legal representatives of all newborns participated in the study.

\section{Results}

During the recruitment phase (November 2017 to July 2020), 219 neonates with PPHN presented at our NICU (12.9\% of total admissions). Eighty-one cases met the study eligibility criteria; however, 37 cases had one or more of the exclusion criteria, and we failed to obtain consent for 16 cases. Ultimately, 28 newborns were successfully randomized into the NebMag $(n=14)$ and IVMag $(n=14)$ groups. There were no deaths or code disclosure for any enrolled case during the 24-h study time frame. The flowchart of study participation is shown in Fig. 1.
Fig. 1 Flowchart of study

enrollment

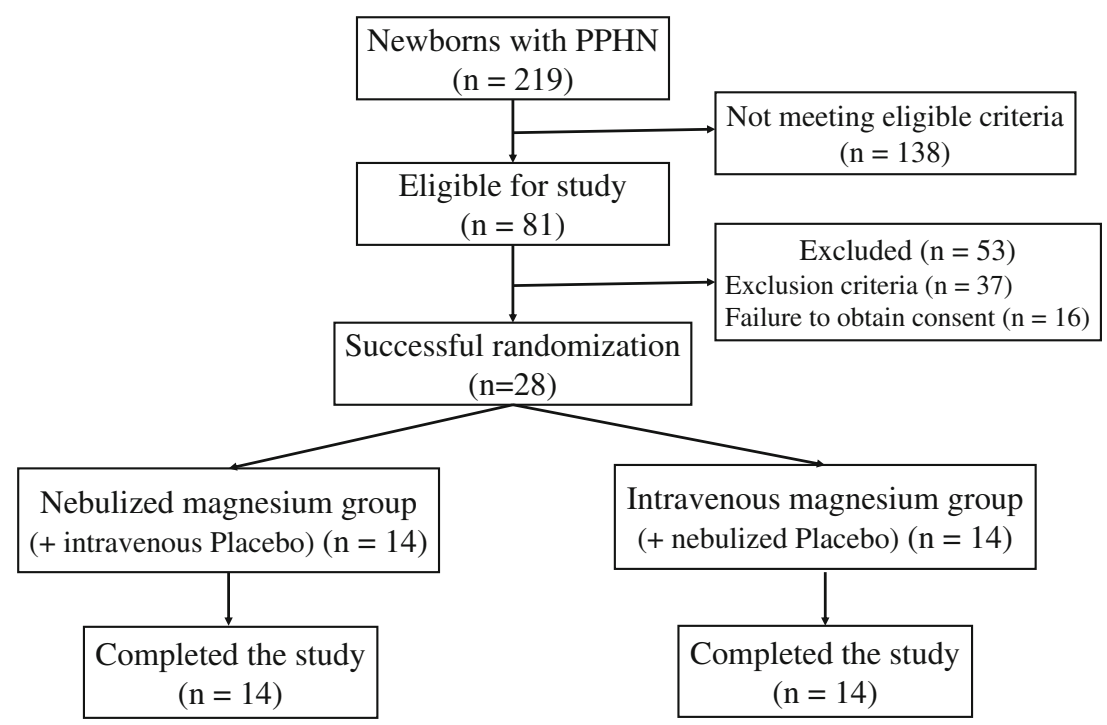


The baseline demographic, ventilatory, and hemodynamic data were comparable between the two groups (Table 1). According to the weight range of $3.1-3.7 \mathrm{~kg}$, the NebMag group received a dose of nebulized magnesium sulfate of approximately $277-330 \mathrm{mg} / \mathrm{kg} / \mathrm{h}$.

Figure 2 depicts the OI changes in both groups over the 24-h study time frame. The OI steadily decreased in both study groups, resulting in a final improvement in OI by $44.3 \%$ and $35.3 \%$ in the NebMag and IVMag groups, respectively (mean difference $-3.14 ; 95 \%$ CI $-5.08,-1.19 ; p$ 0.003 ).

Following study drug administration, the NebMag group had significantly higher MABP and lower VIS measures than that of the IVMag group (Fig. 3). These differences were pronounced as early as $2 \mathrm{~h}$ after study drug administration. At the 24-h study time point, the mean difference in MABP was $2.29 \mathrm{mmHg}$ (95\% CI 1.80, $2.77 ; p 0.000)$ and in VIS was -14.64 (95\% CI -16.52 , $-12.77 ; p 0.000)$.

As shown in Table 1, the baseline mean serum levels of magnesium sulfate were comparable between the two study groups. The mean serum magnesium levels, measured $12 \mathrm{~h}$ after study drug administration, increased from baseline in both groups but were significantly lower in the NebMag group $(1.75 \pm 0.19 \mathrm{mmol} / \mathrm{L})$ than in the IVMag group $(4.02 \pm$ $0.53 \mathrm{mmol} / \mathrm{L}$ ) (mean difference $-2.26 \mathrm{mmol} / \mathrm{L} ; 95 \% \mathrm{CI}$ $-2.58,-1.96 ; p 0.000)$.

There were no observable differences between the two groups in respiratory secretions or episodes of desaturation during the 24-h study time frame.
Table 1 Characteristics and baseline parameters of study participants

\begin{tabular}{|c|c|c|c|}
\hline Variable & $\begin{array}{l}\text { Nebulized magnesium } \\
(\mathrm{n}=14)\end{array}$ & $\begin{array}{l}\text { Intravenous magnesium } \\
(\mathrm{n}=14)\end{array}$ & $\begin{array}{l}p \text { value } \\
\text { (2-tailed) }\end{array}$ \\
\hline Gender (male/female) (n) & $10 / 4$ & $8 / 6$ & $0.695^{\mathrm{a}}$ \\
\hline Gestational age (wk) (mean \pm SD) & $38.7 \pm 1.09$ & $38.9 \pm 1.19$ & $0.556^{\mathrm{b}}$ \\
\hline Birth weight $(\mathrm{kg})($ mean $\pm \mathrm{SD})$ & $3.4 \pm 0.19$ & $3.3 \pm 0.23$ & $0.264^{\mathrm{b}}$ \\
\hline Cesarean section (n) & 11 & 9 & $0.678^{\mathrm{a}}$ \\
\hline Postnatal age, hours (mean $\pm \mathrm{SD}$ ) & $18.5 \pm 5.42$ & $16.5 \pm 4.97$ & $0.318^{\mathrm{b}}$ \\
\hline \multicolumn{4}{|l|}{ Associated condition (n) } \\
\hline Meconium aspiration & 6 & 5 & $1.000^{\mathrm{a}}$ \\
\hline Infection & 3 & 4 & $1.000^{\mathrm{a}}$ \\
\hline Others & 1 & 2 & $1.000^{\mathrm{a}}$ \\
\hline Idiopathic & 4 & 3 & $1.000^{\mathrm{a}}$ \\
\hline \multicolumn{4}{|l|}{ Ventilatory parameters } \\
\hline $\mathrm{PIP}, \mathrm{cm} \mathrm{H}_{2} \mathrm{O}($ mean $\pm \mathrm{SD})$ & $29.6 \pm 2.62$ & $29.4 \pm 2.28$ & $0.876^{\mathrm{b}}$ \\
\hline $\mathrm{PEEP}, \mathrm{cm} \mathrm{H}_{2} \mathrm{O}($ mean $\pm \mathrm{SD})$ & $6.6 \pm 1.09$ & $6.4 \pm 0.85$ & $0.702^{\mathrm{b}}$ \\
\hline $\mathrm{MAP}, \mathrm{cm} \mathrm{H}_{2} \mathrm{O}($ mean $\pm \mathrm{SD})$ & $14.2 \pm 1.40$ & $14.1 \pm 1.25$ & $0.810^{\mathrm{b}}$ \\
\hline Rate, per min $($ mean $\pm \mathrm{SD})$ & $43.9 \pm 2.13$ & $43.6 \pm 2.34$ & $0.676^{\mathrm{b}}$ \\
\hline $\mathrm{FiO}_{2}, \%($ mean $\pm \mathrm{SD})$ & $100 \pm 0.0$ & $100 \pm 0.0$ & $1.000^{\mathrm{b}}$ \\
\hline \multicolumn{4}{|l|}{ Arterial blood gases } \\
\hline $\mathrm{pH}($ mean $\pm \mathrm{SD})$ & $7.24 \pm 0.04$ & $7.26 \pm 0.05$ & $0.340^{\mathrm{b}}$ \\
\hline $\mathrm{PaO}_{2}, \mathrm{mmHg}($ mean $\pm \mathrm{SD})$ & $38.8 \pm 1.72$ & $38.6 \pm 1.95$ & $0.760^{\mathrm{b}}$ \\
\hline $\mathrm{PaCO}_{2}, \mathrm{mmHg}($ mean $\pm \mathrm{SD})$ & $41.6 \pm 3.59$ & $40.9 \pm 2.59$ & $0.551^{\mathrm{b}}$ \\
\hline $\mathrm{HCO}_{3}, \mathrm{mmol} / \mathrm{L}($ mean $\pm \mathrm{SD})$ & $16.9 \pm 0.86$ & $17.5 \pm 2.91$ & $0.520^{\mathrm{b}}$ \\
\hline Oxygenation Index $($ mean \pm SD) & $36.8 \pm 4.34$ & $36.5 \pm 2.15$ & $0.823^{\mathrm{b}}$ \\
\hline $\mathrm{EPAP}, \mathrm{mmHg}($ mean $\pm \mathrm{SD})$ & $59.4 \pm 2.17$ & $58.9 \pm 2.60$ & $0.534^{\mathrm{b}}$ \\
\hline $\mathrm{MABP}, \operatorname{mmHg}($ mean $\pm \mathrm{SD})$ & $44.8 \pm 1.12$ & $45.5 \pm 1.38$ & $0.303^{\mathrm{b}}$ \\
\hline Vasoactive inotrope score (mean \pm SD) & $26.1 \pm 2.13$ & $26.2 \pm 2.90$ & $0.990^{\mathrm{b}}$ \\
\hline Serum magnesium, mmol/L (mean $\pm \mathrm{SD})$ & $0.89 \pm 0.073$ & $0.91 \pm 0.067$ & $0.852^{\mathrm{b}}$ \\
\hline
\end{tabular}

EPAP Estimated pulmonary artery pressure; $\mathrm{FiO}_{2}$ Fraction of inspired oxygen; $M A B P$ Mean arterial blood pressure; MAP Mean airway pressure; $P E E P$ Positive end-expiratory pressure; PIP Peak inspiratory pressure

${ }^{\mathrm{a}}$ Fisher exact test

${ }^{\mathrm{b}}$ Student t-test 
Fig. 2 Changes in oxygenation index (OI). Following study drug administration, the nebulized magnesium group had lower measures of OI than the intravenous magnesium group. The difference in OI between groups increased by time and became statistically significant only at the 24-h study time point (2-h, $p=0.458 ; 6-\mathrm{h} p=0.176 ; 12$ h, $p=0.051 ; 24-\mathrm{h}, p=0.003$ )

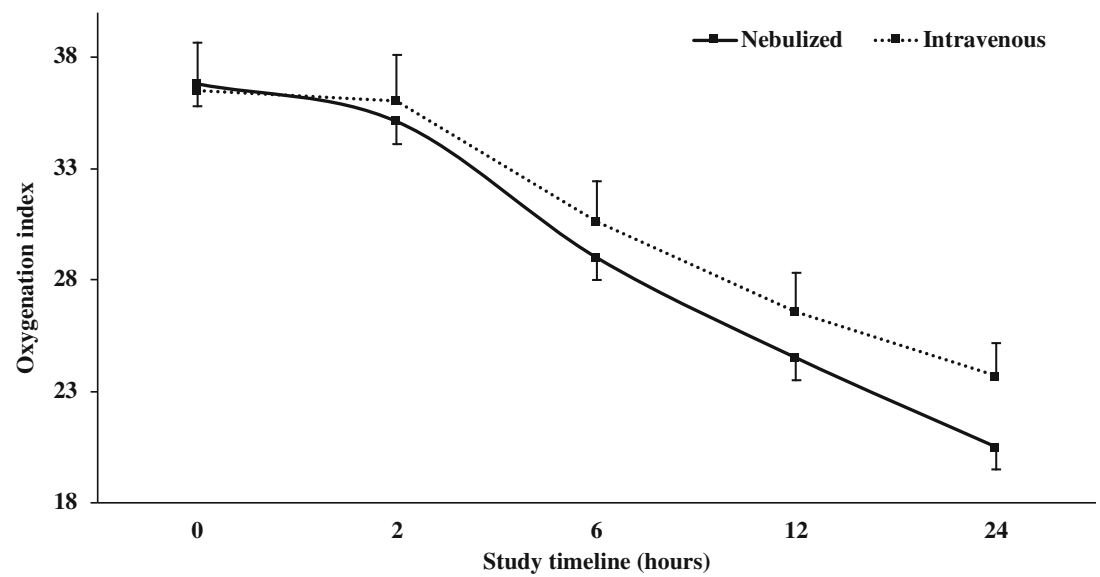

\section{Discussion}

To the authors' knowledge, this is the first clinical trial to investigate the effectiveness of nebulized magnesium sulfate in PPHN. The authors found that neonates with PPHN who received nebulized magnesium for $24 \mathrm{~h}$ had an improvement in OI by $44.3 \%$, compared with $35.3 \%$ in those received intravenous magnesium. Moreover, the nebulized magnesium group had a higher MABP, received lower doses of inotropes, and had a less increase in serum magnesium levels than that of the intravenous magnesium group. Therefore, nebulized magnesium may be an effective therapeutic modality for PPHN, but larger studies are required to confirm this information.

Delivering magnesium by nebulization can enhance its effectiveness and safety. The direct drug deposition to the lung may achieve higher concentrations in the pulmonary interstitial space and vasculature at even lower levels in the systemic circulation. This minimizes the risk of systemic hypotension, making nebulized magnesium act as a "selective" pulmonary vasodilator. Moreover, the potential for preferential distribution of nebulized particles to more ventilated lung areas, where it dilates blood vessels and enhances blood flow-"micro-selectivity", may optimize ventilation/perfusion

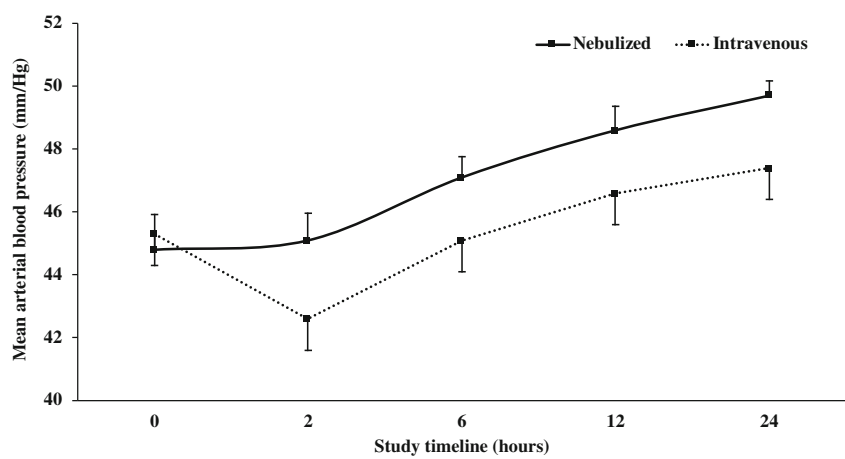

Fig. 3 Changes in mean arterial blood pressure and vasoactive inotropic score. Following study drug administration, the nebulized magnesium group had higher mean arterial blood pressure (MABP) and lower matching and improves oxygenation. In contrast, systemically administered magnesium may impair gas exchange due to nonselective pulmonary vasodilation and increased blood flow to poorly ventilated lung areas [12, 15-17].

However, delivering adequate amounts of magnesium by nebulization is challenged by concentration and volume limits. Increasing magnesium concentration is limited to the maximum accepted tonicity $(500 \mathrm{mOsm} / \mathrm{L})$ of nebulized solutions, above which there is an increased risk of bronchoconstriction and airway irritation [16-18]. On the other side, increasing the volume is limited by the nebulization abilities of current devices $[17,19]$. Considering these limits, the dose of nebulized magnesium in our study was $1024 \mathrm{mg} / \mathrm{h}$ using isotonic magnesium solution and the available jet nebulizer $(4-\mathrm{mL} / \mathrm{h})$ at the authors' NICU. Some previous studies on asthmatic adults and older children used even higher doses of nebulized magnesium sulfate, up to $1500 \mathrm{mg}$ over an hour $[19,20]$. However, the doses of nebulized magnesium in the present study $(277-330 \mathrm{mg} / \mathrm{kg} / \mathrm{h})$, are much higher, on a weight-equivalent basis, than that used in previous studies (e.g., in bronchial asthma, bronchiolitis) [19-22]. Such higher weight-equivalent doses may explain, at least in part, the effectiveness of nebulized magnesium in treating PPHN in this

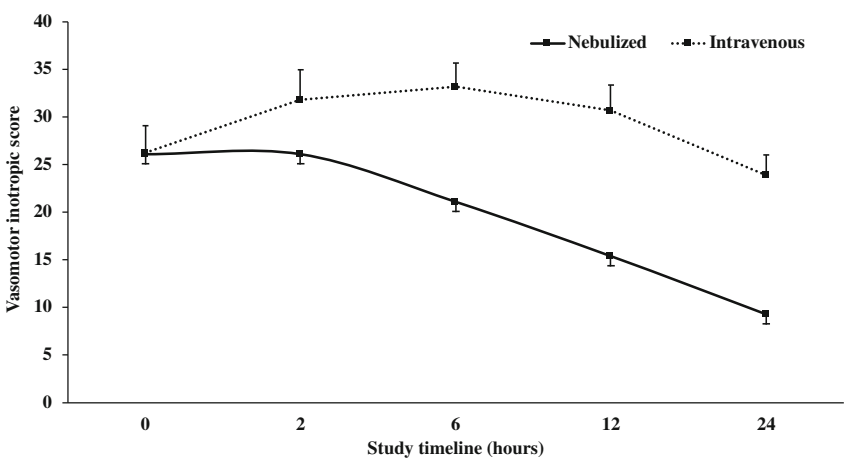

vasoactive inotropic score (VIS) than the intravenous magnesium group. The differences in MABP and VIS between the two groups were statistically significant $(p=0.000)$ at the 2-, 6-, 12-, and 24-h study time points 
study, compared with the limited effectiveness of nebulized magnesium, in treating older children with bronchiolitis and bronchial asthma.

Although the present study used relatively high weightequivalent doses of nebulized magnesium, the authors could not ascertain the exact fraction of nebulized drug that actually reached the alveolar space of newborns. Scintigraphic studies demonstrate that the amount of nebulized drug that reaches alveolar space during mechanical ventilation is $<10 \%-20 \%$ of the volume placed in the reservoir of nebulizer; this may be even lower in neonates with relatively larger dead spaces and those not connected to mechanical ventilation. Therefore, nebulized magnesium sulfate may have limited effectiveness in spontaneously breathing neonates with mild PPHN [23-25]. Delivery of nebulized drugs during mechanical ventilation is technically complex and affected by numerous variables related to solution properties, nebulizer, humidification and heating systems, breathing circuit and artificial airways, ventilator settings, patient, and lung anatomy and pathology [17, $23,25,26]$. Having an efficient and consistent nebulization system during mechanical ventilation remains technically challenging. Using a vibrating-mesh nebulizer, synchronizing nebulization with inspiratory airflow, and minimizing interruptions (e.g., using a dual-chamber volumetric infusion system) may increase the amount of nebulized drug that reach alveolar spaces $[17,26]$.

In the present study, the nebulizer was operated by pressurized medical air from the ventilator. This is expected to reduce the $\mathrm{FiO}_{2}$ delivered to patients relative to that set on the ventilator by approximately $10 \%$. Indeed, the authors didn't compensate for that in calculating OI, which might result in inappropriately higher OI values after starting the study. On the other hand, the evaporative effect of the dry air used to run the jet nebulizer might increase the solution tonicity in the reservoir during nebulization [25]. This potential osmolarity change could be avoided in future studies by using a vibrating-mesh nebulizer, in which the fluid in the reservoir is not exposed to air until nebulized $[16,17]$.

In the present study, the authors did not repeat echocardiography at a consistent study time point to assess the effects of treatment on pulmonary artery pressure, right-to-left shunting, and cardiac function; this was not applicable at the present setting. However, all enrolled infants had an echocardiogram before the study to confirm the diagnosis of PPHN and exclude structural heart diseases other than PDA and foramen ovale, and follow-up echocardiograms were performed thereafter but at variable time points. Instead, we used OI as an objective indicator of the oxygenation, severity of PPHN, and response to therapy during the study duration [1].

The authors acknowledge that this is a pilot study with some limitations related to the small sample size, narrow scope, and limited study time frame. First, they included a specific set of newborns with PPHN and associated conditions. Important categories, such as preterm \pm respiratory distress syndrome, less severe cases $(\mathrm{OI}<30$, not connected to ventilators), and newborns with congenital heart defect or other anomalies (e.g., congenital diaphragmatic hernia) have to be addressed in future studies. Second, the study used only one dosage of magnesium by continuous nebulization. Questions on the effects of using different doses or intermittent nebulization remain unanswered. Third, the authors did not study the combined effects of nebulized magnesium sulfate and other pulmonary vasodilators, such as iNO, prostanoids, and phosphodiesterase inhibitors. Last, the limited study time frame did not allow to address important outcomes, such as ventilation time, length of NICU stay, survival rate, and long-term neurodevelopment.

\section{Conclusions}

Nebulized magnesium may be an effective therapeutic modality for neonates with severe PPHN on mechanical ventilation, but larger studies are required to confirm this information.

Acknowledgments The authors would like to express their gratitude to all physicians and nurses at the NICU of Sohag University Hospital for their dedicative clinical management and care of newborns included in the study.

Authors' Contributions EA initiated the research idea. All authors contributed to the study conception and design. EA and SMM shared in data collection. MOA designed the pharmaceutical preparations. EA wrote the first draft of the manuscript. MAEA supervised the whole study. All authors commented on and critically revised previous versions of the manuscript. All authors read and approved the final manuscript. MAEA will act as guarantor for this paper.

Data Availability Anonymous patient's data are available upon reasonable request through contacting the corresponding author.

\section{Compliance with Ethical Standards}

Ethics Approval This study was approved by the Research Ethics Committee of Faculty of Medicine, Sohag University (Egypt), and was carried out following the principles contained within the 1964 Declaration of Helsinki and as revised in 2013.

Conflict of Interest None.

Consent for Publication Informed consent was obtained from parents or authorized legal representatives of all newborns who participated in the study for publication of anonymous patients' data.

\section{References}

1. Mathew B, Lakshminrusimha S. Persistent pulmonary hypertension in the newborn. Children. 2017;4(8):63. 
2. Krishna MR, Kumar RK. Diagnosis and management of critical congenital heart diseases in the newborn. Indian J Pediatr. 2020;87(5):365-71.

3. Lakshminrusimha S, Keszler M. Persistent pulmonary hypertension of the newborn. Neorev. 2015;16(12):e680-92.

4. El-Khuffash A, McNamara PJ, Breatnach C, et al. The use of milrinone in neonates with persistent pulmonary hypertension of the newborn - a randomised controlled trial pilot study (MINT 1): study protocol and review of literature. Matern Heal Neonatol Perinatol. 2018;4(1):24.

5. Lai M-Y, Chu S-M, Lakshminrusimha S, Lin H-C. Beyond the inhaled nitric oxide in persistent pulmonary hypertension of the newborn. Pediatr Neonatol. 2018;59(1):15-23.

6. Wedgwood S, Steinhorn RH, Lakshminrusimha S. Optimal oxygenation and role of free radicals in PPHN. Free Radic Biol Med. 2019;142:97-106.

7. Nakwan N. The practical challenges of diagnosis and treatment options in persistent pulmonary hypertension of the newborn: a developing country's perspective. Am J Perinatol. 2018;35(14): 1366-75.

8. Chettri S, Bhat BV, Adhisivam B. Current concepts in the management of meconium aspiration syndrome. Indian J Pediatr. 2016;83(10):1125-30.

9. Kelly LE, Ohlsson A, Shah PS. Sildenafil for pulmonary hypertension in neonates. Cochrane Database Syst Rev. 2017;8(8): CD005494.

10. Shivanna B, Gowda S, Welty SE, Barrington KJ, Pammi M. Prostanoids and their analogues for the treatment of pulmonary hypertension in neonates. Cochrane Database Syst Rev. 2019;10(10):CD012963.

11. Ho JJ, Rasa G. Magnesium sulfate for persistent pulmonary hypertension of the newborn. Cochrane Database Syst Rev. 2007;18(3): CD005588.

12. Shaltout F, Hegazy R, Aboulghara H, Abdel ML. Magnesium sulphate versus sildenafil in the treatment of persistent pulmonary hypertension of the newborn. Int J Clin Pediatr. 2012;1(1):19-24.

13. de Boode WP, Singh Y, Molnar Z, et al. Application of neonatologist performed echocardiography in the assessment and management of persistent pulmonary hypertension of the newborn. Pediatr Res. 2018;84(S1):68-77.

14. Gaies MG, Gurney JG, Yen AH, et al. Vasoactive-inotropic score as a predictor of morbidity and mortality in infants after cardiopulmonary bypass. Pediatr Crit Care Med. 2010;11(2):234-8.
15. Hill NS, Preston IR, Roberts KE. Inhaled therapies for pulmonary hypertension. Respir Care. 2015;60(6):794-805.

16. Coates AL, Leung K, Vecellio L, Schuh S. Testing of nebulizers for delivering magnesium sulfate to pediatric asthma patients in the emergency department. Respir Care. 2011;56(3):314-8.

17. Dhand R. How should aerosols be delivered during invasive mechanical ventilation? Respir Care. 2017;62(10):1343-67.

18. Turker S, Dogru M, Yildiz F, Yilmaz SB. The effect of nebulised magnesium sulphate in the management of childhood moderate asthma exacerbations as adjuvant treatment. Allergol Immunopathol (Madr). 2017;45(2):115-20.

19. Bessmertny O, DiGregorio RV, Cohen H, et al. A randomized clinical trial of nebulized magnesium sulfate in addition to albuterol in the treatment of acute mild-to-moderate asthma exacerbations in adults. Ann Emerg Med. 2002;39(6):585-91.

20. Aggarwal P, Sharad S, Handa R, Dwiwedi SN, Irshad M. Comparison of nebulised magnesium sulphate and salbutamol combined with salbutamol alone in the treatment of acute bronchial asthma: a randomised study. Emerg Med J. 2006;23(5):358-62.

21. Knightly R, Milan SJ, Hughes R, et al. Inhaled magnesium sulfate in the treatment of acute asthma. Cochrane Database Syst Rev. 2017;11(11):CD003898.

22. Modaresi MR, Faghihinia J, Kelishadi R, et al. Nebulized magnesium sulfate in acute bronchiolitis: a randomized controlled trial. Indian J Pediatr. 2015;82(9):794-8.

23. Dugernier J, Ehrmann S, Sottiaux T, et al. Aerosol delivery during invasive mechanical ventilation: a systematic review. Crit Care. 2017;21(1):264.

24. Cosa N, Costa E Jr. Inhaled pulmonary vasodilators for persistent pulmonary hypertension of the newborn: safety issues relating to drug administration and delivery devices. Med Devices (Auckl). 2016;9:45-51.

25. Davis MD, Donn SM, Ward RM. Administration of inhaled pulmonary vasodilators to the mechanically ventilated neonatal patient. Pediatr Drugs. 2017;19(3):183-92.

26. Berlinski A, Willis JR. Effect of tidal volume and nebulizer type and position on albuterol delivery in a pediatric model of mechanical ventilation. Respir Care. 2015;60(10):1424-30.

Publisher's Note Springer Nature remains neutral with regard to jurisdictional claims in published maps and institutional affiliations. 